\title{
An Improved Density Reinitialization Method for Dam Break Simulation with SPH
}

\author{
Song Chai ${ }^{1,2, a}$, Guoping Miao ${ }^{1}$ \\ ${ }^{1}$ State Key Laboratory of Ocean Engineering, School of Naval Architecture, Ocean and Civil \\ Engineering, Shanghai Jiao Tong University, Shanghai 200240, China \\ ${ }^{2}$ Wison (Nantong) Heavy Industry Co., Ltd., Nantong, Jiangsu 226009, China \\ a chaisong@wison.com
}

\begin{abstract}
Keywords: density reinitialization; dam break; smoothed particle hydrodynamics; pressure oscillation; self-adaptive

Abstract. In this work, the realization of density reinitialization with Smoothed Particle Hydrodynamics (SPH) techniques is examined. A self-adaptive density reinitialization method is proposed. Instead of using fixed step intervals for reinitialization, this method obtains oscillation of density field by introducing two variables for measuring the density variation acceleration and defining the density variation criteria; and then performs density reinitialization once oscillation exceeds the criteria which is self-adjusted according to the density field. Numerical test on two-dimension dam break illustrates that this method is able to adjust the reinitialization frequency according to density field oscillation during SPH simulation, while achieving good accuracy.
\end{abstract}

\section{Introduction}

Originally suggested in an astrophysical context [1] [2], Smoothed Particle Hydrodynamics (SPH) is a completely mesh-free, fully conservative hydrodynamics method.

SPH has long been appreciated for its highly desirable properties in the hydrodynamics simulation, one of which is SPH's natural adaptivity that comes without the burden of additional infrastructure such as an adaptive mesh. Probably, the most outstanding advantage of SPH is the direct integration of the conservation of mass, energy and momentum into discrete SPH formulations so that the independent of numerical resolution is guaranteed.

The numerical results from SPH simulations are generally realistic, however large pressure oscillations may happen in the pressure field of the particles, especially for the nonlinear problem in hydrodynamics. Several approaches have been developed to overcome this problem including correcting the kernel [3] and integrating an incompressible solver. One of the most straight forward and computationally least expensive is to perform a filter over the density of the particles and re-assign a density to each particle [4].

In practice such filter is to be performed at fixed step intervals, however, as the oscillation of density field is normally non-linear, such method cannot obtain good accuracy with high computational efficiency for hydrodynamic problem. The major challenge in this respect is to design a filtering scheme which can apply the density reinitialization according to the oscillation of density field. In this way, the density reinitialization frequency can be self-adaptive and automatically adjusted to the density field.

\section{SPH methodology}

As a basis for later discussion, only a brief overview of SPH methodology is provided here.

The main features of the SPH method are described in detail in the following reviews [5-10]. At the heart of SPH is the smooth representation of any function by using an interpolating kernel, $W$. Within the kernel definition, $h$ is the smoothing length, controls the size of the area where the contribution from the rest of the particles must be estimated. In addition, the kernel function should meet several requirements including positivity, compact support and normalization. Detailed discussion about the kernel function can be found in the literature[8, 9, 11]. The two-dimensional 
basic conservation equations can be represented in SPH notation following[8], while particles were moved by using the so-called XSPH[12].

\subsection{Kernel function}

The so-called Wendland functions[13] are used as kernel function here, as these kernels are not prone to the pairing instability which has been discussed in detail in[14]. In the following numerical tests the C2 smooth in 2-dimension is applied

$$
W(r, h)=\frac{7}{4 \pi h^{2}}\left(1-\frac{q}{2}\right)^{4}(1+2 q) \quad 0 \leq q \leq 2 .
$$

where $q=r / h, r$ being the distance between pairing particles.

\subsection{Time step criteria}

A variable time step scheme is used according to Monaghan J. and Kos A.[15]:

$$
\delta t=0.3 \min \left(\min _{a}\left(\sqrt{\frac{h}{\left|f_{a}\right|}}\right), \min _{a}\left(\frac{h}{c_{s}+\max _{b}\left|h \vec{v}_{a b} \vec{r}_{a b} / \vec{r}_{a b}^{2}\right|}\right)\right) .
$$

where $\left|f_{a}\right|$ is the force per unit mass, $c_{s}$ is the speed of sound.

\subsection{Equation of state}

By using an equation of state, the incompressibility condition is approximated with the density variation $\Delta \rho<0.01 \rho$. Following[15, 16], the relation between pressure and density follows the expression

$$
P=B\left[\left(\frac{\rho}{\rho_{0}}\right)^{\gamma}-1\right] .
$$

where $\gamma=7$ and $B=c_{0}^{2} \rho_{0} / \gamma, \rho_{0}$ is the reference density and $c_{0}=\left.\sqrt{(\partial P / \partial \rho)}\right|_{\rho_{0}}$ is the speed of sound at the reference density.

\subsection{Density correction}

The Moving Least Squares (MLS) approach, developed by Dilts G. A. [17] and applied successfully by Colagrossi A. and Panizzo A. [4] [18], is applied. The density field will be corrected by

$$
\rho_{a}^{\text {new }}=\sum_{b} \rho_{b} \widetilde{W}_{a b} \frac{m_{b}}{\rho_{b}}=\sum_{b} m_{b} \widetilde{W}_{a b} .
$$

where, the detailed correction kernel $\widetilde{W}_{a b}$ can be found in[4].

\section{Self-adaptive density reinitialization}

To assess the density estimation accuracy, a simple method can be performed. In incompressible fluid problems, the fluid density will stay unchanged to its theoretical value $\rho_{0}$. So the relative deviation $\varepsilon$ between the estimated and theoretical density value can be measured as

$$
\varepsilon=\log _{10}\left(\left(\sum_{i=1}^{N}\left|\rho_{i}-\rho_{0}\right|\right) / N \rho_{0}\right),
$$


where $N$ is the number of fluid particles, $\rho_{i}$ is the density of the $i^{\text {th }}$ fluid particle, $\rho_{0}$ is the density of the fluid in research (for water, $\rho_{0}=1000 \mathrm{~kg} / \mathrm{m}^{3}$ ). In practice, $\varepsilon$ is calculated in every time step by

$$
\varepsilon(i t)=\log _{10}\left(\left(\sum_{i=1}^{N}\left|\rho_{i}(i t)-\rho_{0}\right|\right) / N \rho_{0}\right),
$$

where it is the time step.

The basic concept is to apply the density reinitialization when density field oscillation is significant. So it is critical to find an appropriate way to evaluate whether it is needed to perform reinitialization on density field. For this purpose, two variables are imposed here: density variation acceleration $a(i t)$ and density oscillation limit $L(i t)$.

The density oscillation limit is defined as

$$
L(i t)=\min (0.05 \times|\varepsilon(1)|, 0.05 \times|\varepsilon(i t)|) /(\Delta i t)^{2},
$$

where $\Delta i t$ is the step interval between the last time step it ${ }^{*}$ when density reinitialization performed and the current time step it, which can be calculated by

$$
\left\{\begin{array}{ll}
\Delta i t=1 & i t=1 \\
\Delta i t=i t-i t^{*} & \text { it }>1
\end{array},\right.
$$

The density variation acceleration is defined as

$$
a(i t)=\left|\varepsilon(i t)-\varepsilon\left(i t^{*}\right)\right| \times(\Delta i t)^{-3 / 2},
$$

Both $a(i t)$ and $L(i t)$ are related with $\varepsilon(i t)$ and $\Delta i t$, which means they will update in every time step to obtain the current density field oscillation and compare with the accordingly adjusted criteria.

In each time step, these two variables are calculated and compared with each other. If $a(i t)>L(i t)$, the density reinitialization will be performed and the current time step will be recorded as it*; if not, the reinitialization will be skipped.

\section{Case study}

\subsection{Dam break in 2-dimension}

When the vertical end wall holding a body of water above a horizontal bottom is quickly removed, the resulting flow of water is called dam break problem.

To simulate such problem with SPH method, fluid particles were initially placed in a staggered $0.03 \times 0.03 \mathrm{~m}$ grid in a $4 \times 4 \mathrm{~m}$ box, while the fluid particles fill an $1 \times 2 \mathrm{~m}$ area in the box as shown in Fig. 1(a). In such configuration, water will rebound between the two side walls several times in a relatively short period, during which the density variation can be violent and the performance of the proposed self-adaptive method can be assessed.

Each numerical experiment is performed for 10 seconds, after which the flow becomes nearly still as there is no other external force, see Fig. 1(b).

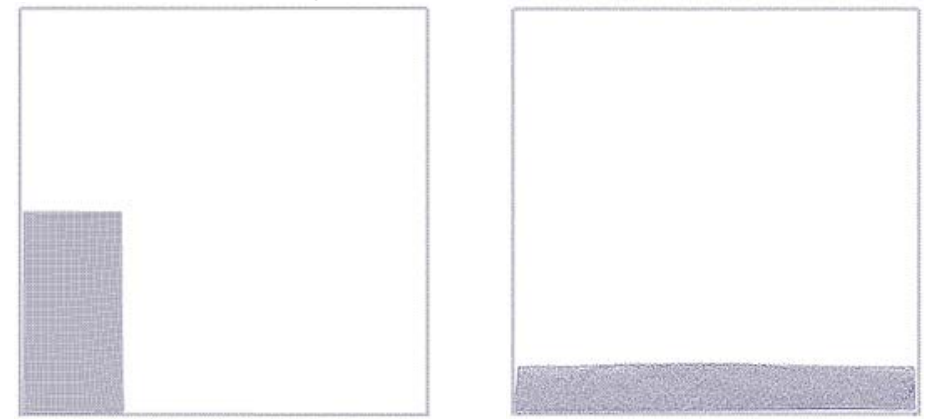

Figure 1(a). Dam break simulation at $\mathrm{t}=0 \mathrm{~s}$; (b). Dam break simulation at $\mathrm{t}=10 \mathrm{~s}$ 


\subsection{Results and discussion}

Normally, the density reinitialization will be performed in every 30 time steps to achieve a more stable density field. For self-adaptive method, the times of reinitialization is not able to be estimated in advance. However, in order to give a more comprehensive comparison result, the times of reinitialization by self-adaptive method is recorded and then the fixed step intervals is estimated to be 93 to perform nearly the same times of reinitialization. As a result, these three density reinitialization schemes are performed separately in numerical experiments with SPH.

The results of relative deviation are shown in Fig. 2, Fig. 3 and Fig. 4, respectively for Case 1 (step intervals $=30$ ), Case 2 (step intervals $=93$ ) and Case 3 (self-adaptive method).

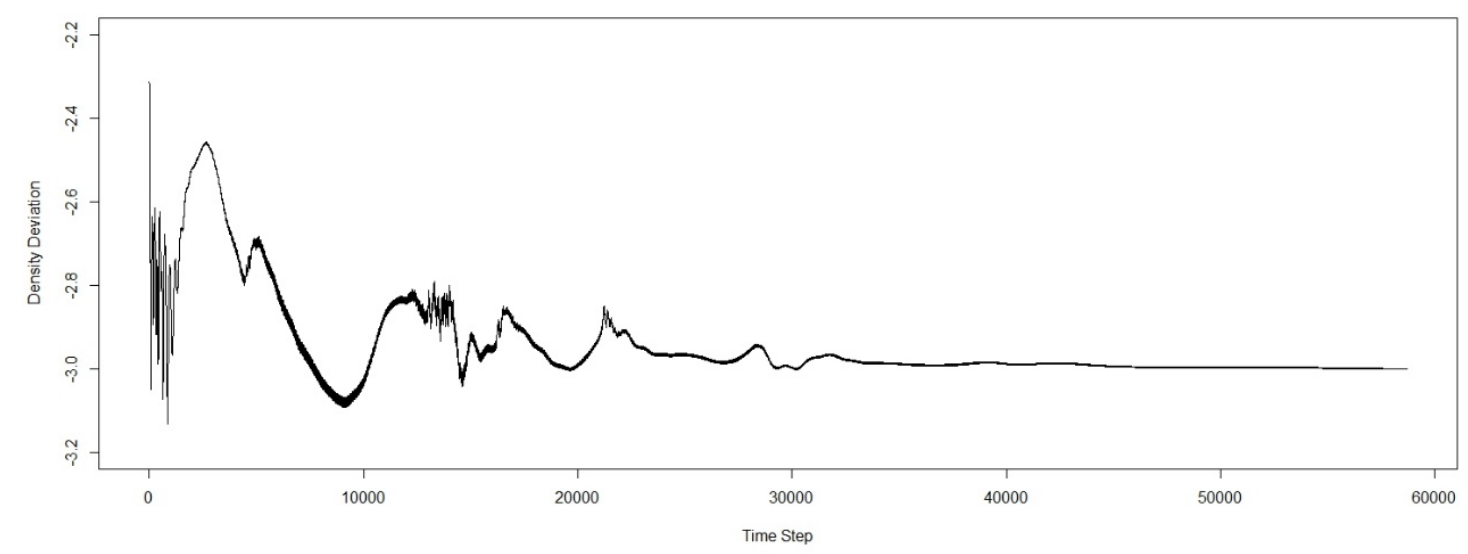

Figure 2. Relative density deviation of case 1 (step intervals $=30$ )

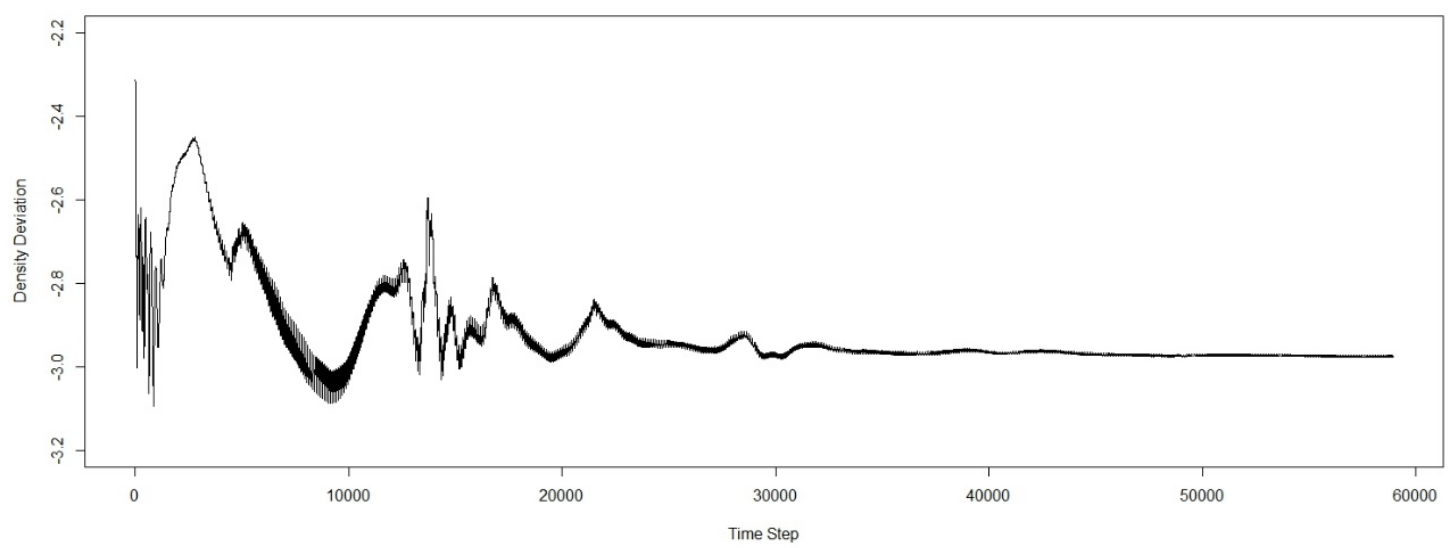

Figure 3. Relative density deviation of case 2 (step intervals $=93$ )

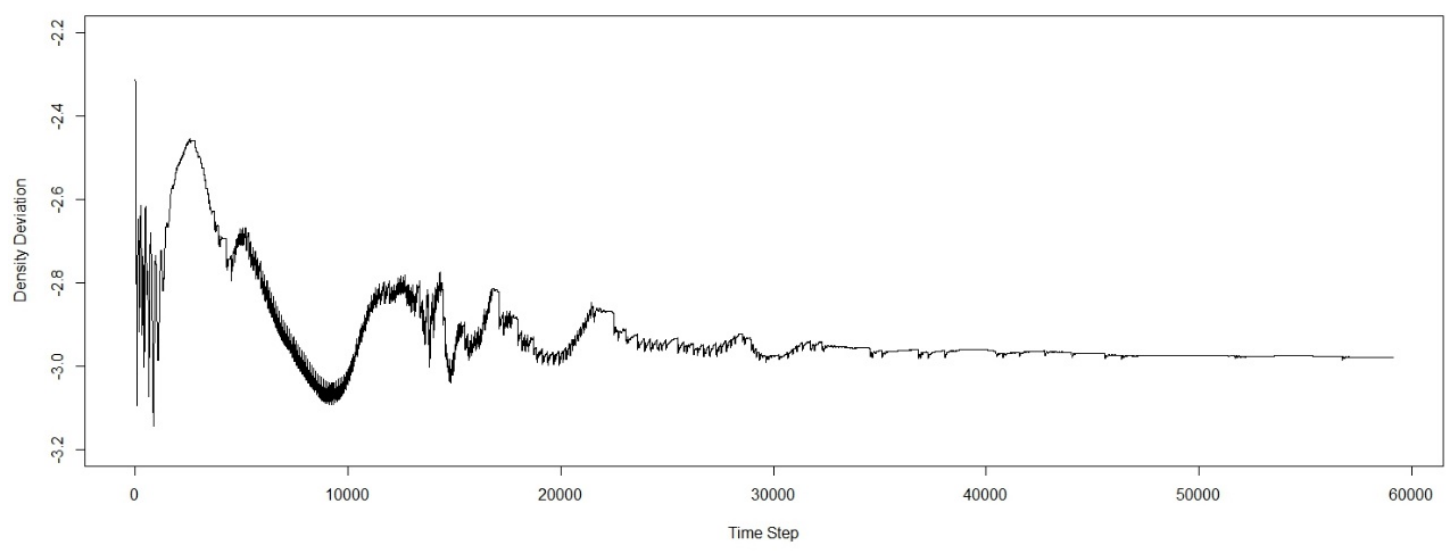

Figure 4. Relative density deviation of case 3 (self-adaptive method)

The oscillation of density field in the first stage of dam break is the largest. During this period, the results shown in all three cases are almost the same. However, after about 5000 time steps, there shows different performances on density field. From 5000 to 20000 time steps, the oscillation in Fig. 
2 (Case 1) is the smallest, which can be foreseen as generally the more frequently the density reinitialization is performed, the less oscillation exists. It becomes more significant when the fixed steps interval changes from 30 to 93, with larger density field oscillation can be seen in Fig. 3 (Case 2). After 20000 time steps, the density field becomes more stable as the water flow tends to become still.

To clearly show the different performance between all three cases, relative density deviation from 5000 to 20000 time step are extracted, see Fig. 5, Fig. 6 and Fig. 7, respectively for Case 1 (step intervals $=30$ ), Case 2 (step intervals $=93$ ) and Case 3 (self-adaptive method).

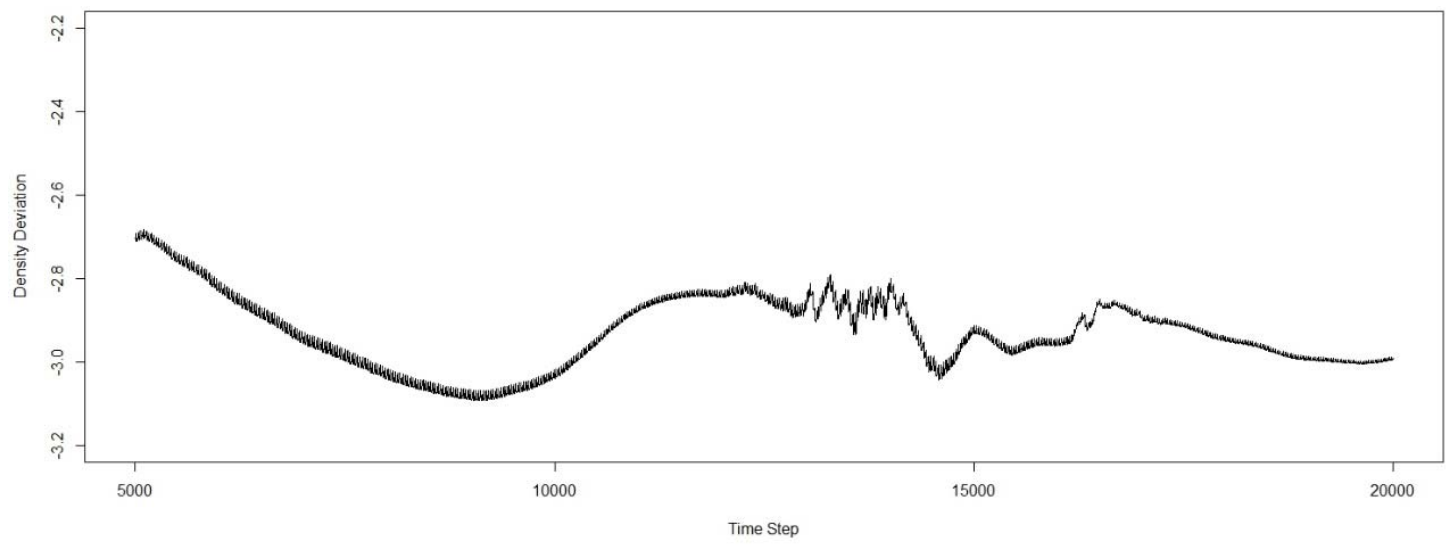

Figure 5. Relative density deviation of case 1 (5000 20000, step intervals $=30)$

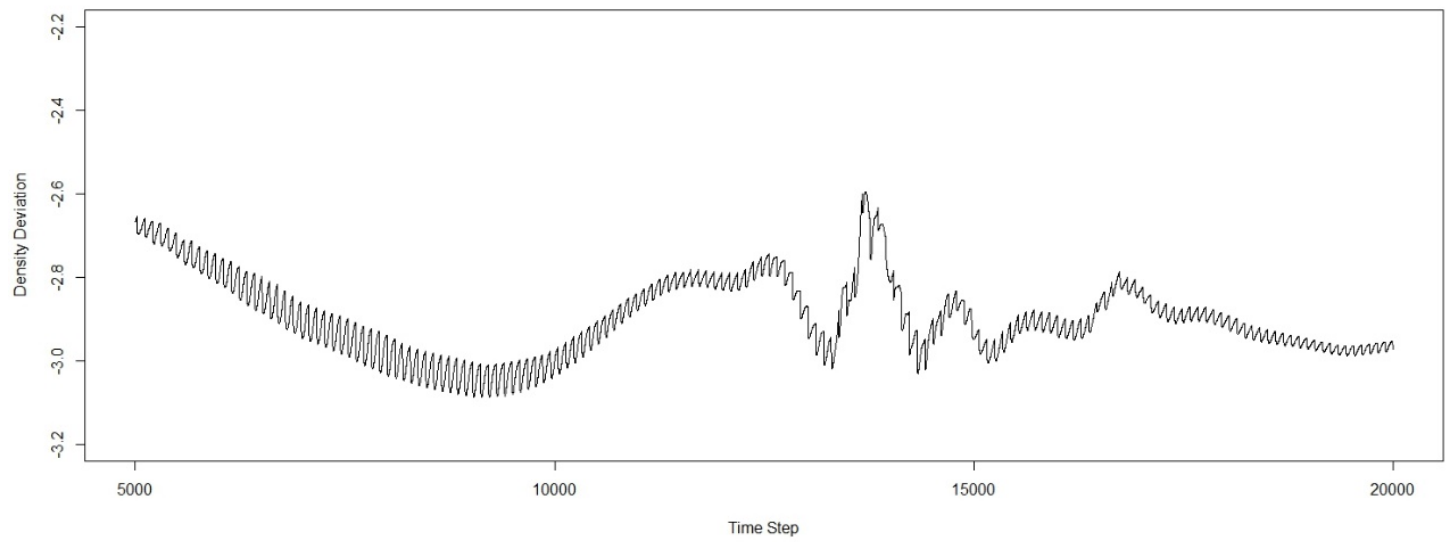

Figure 6. Relative density deviation of case 2 (5000 20000, step intervals $=93$ )

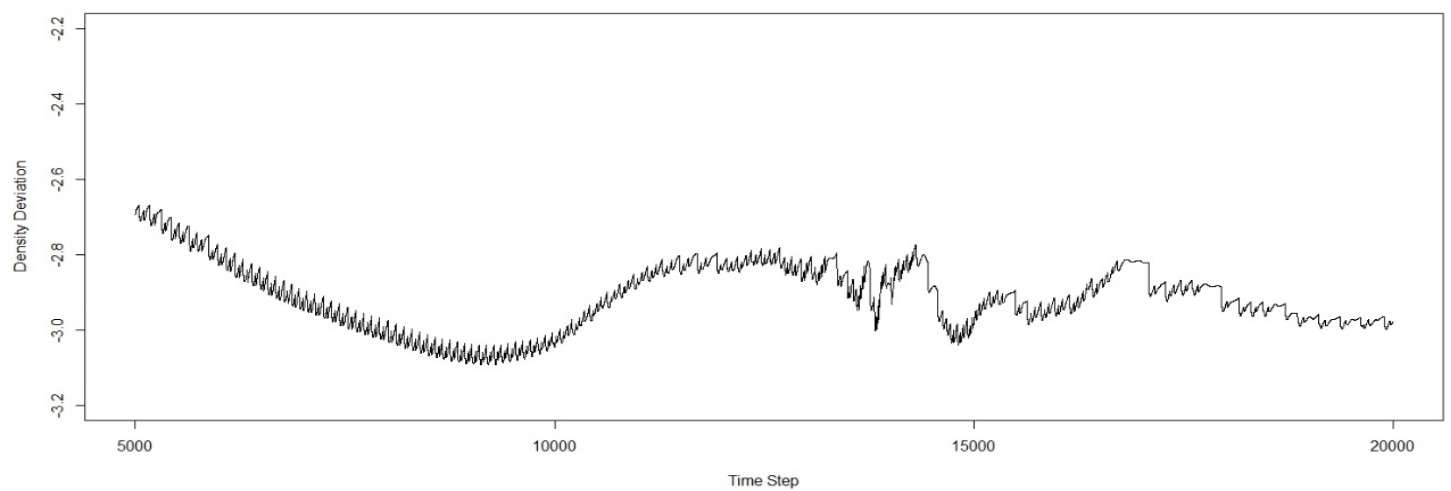

Figure 7. Relative density deviation of case 3 (5000 20000, self-adaptive method)

For 10 seconds numerical simulation, the total time steps are near 60000. As shown in Table 1, the density reinitialization has been performed for 1956 times in Case 1, while in Case 3 the total times have been reduced to 633 which is nearly one third of that in case 1. Compared with Case 1 and Case 2, in Case 3 the times of reinitialization performed before 20000 time steps is much more than that after 20000 time steps, see Table 1. This result clearly illustrates that, with fixed steps interval 
method, the times of reinitialization is proportional to overall time steps; however, with self-adaptive method, the times of reinitialization depends on the density oscillation. This is just the outstanding advantage of the proposed method - the reinitialization frequency can be self-adaptively adjusted according to the density oscillation.

Table 1. Times of reinitialization performed

\begin{tabular}{cccc}
\hline Time Step Range & Case 1 & Case 2 & Case 3 \\
\hline Over all time steps & 1956 & 633 & 629 \\
\hline Before 20000 time steps & 666 & 215 & 538 \\
\hline After 20000 time steps & 1290 & 418 & 91 \\
\hline
\end{tabular}

To assess the accuracy performance, mean value and standard deviation of relative deviation of all time steps are calculated, see Table 2.

Table 2. Relative density deviation statistic

\begin{tabular}{cccc}
\hline & Case 1 & Case 2 & Case 3 \\
\hline \multirow{2}{*}{ Mean Value of $\varepsilon$} & -2.943 & -2.918 & -2.923 \\
& 1 & 7 & 8 \\
\hline Standard deviation of $\varepsilon$ & 0.1083 & 0.1062 & 0.1051 \\
\hline
\end{tabular}

The mean value of relative deviation in Case 1 is the smallest as the more reinitialization performed the better accuracy can be achieved. Compared with Case 2, which performed almost the same times of reinitialization, the mean value of relative deviation in Case 3 is smaller. It is clear that the self-adaptive method achieves better accuracy within the same level of reinitialization performed, while its accuracy performance is still limited to the total times of reinitialization.

The standard deviation of from the three Cases are slightly different, but the smallest value in Case 3 may illustrate that self-adaptive method has the best performance on the stability of density field.

\section{Conclusion}

A new method to perform reinitialization on density field in SPH simulation is proposed in this paper. At the heart of this method, two variables are introduced to define the density variation acceleration and reinitialization criteria, so that the oscillation of density field in every time step can be measured and checked, and the reinitialization frequency can be automatically adjusted accordingly. This so-called self-adaptive method is tested with numerical simulation of two-dimension dam break problem. The numerical results proved that: the times to perform reinitialization can be significantly reduced while obtaining almost the same accuracy; and the reinitialization was performed more frequently when the density oscillation became significant, which means that, the reinitialization frequency was self-adjusted according to the oscillation of density field.

However, as the density deviation is obtained from all fluid particles, this method ignores the different density oscillation of particles from different area, e.g. particles of free surface usually presents the most violent motion. Further research may focus on the free surface particles to obtain more calculation efficiency and more stable density field.

\section{References}

[1] Lucy, L., 1977, "A numerical approach to the testing of the fission hypothesis," The astronomical journal, 82, pp. 1013-1024.

[2] Gingold, R. A., and Monaghan, J. J., 1977, "Smoothed particle hydrodynamics: theory and application to non-spherical stars," Monthly Notices of the Royal Astronomical Society, 181(3), pp. 375-389. 
[3] Bonet, J., and Lok, T. S. L., 1999, "Variational and momentum preservation aspects of Smooth Particle Hydrodynamic formulations," Computer Methods in Applied Mechanics and Engineering, 180(1-2), pp. 97-115.

[4] Colagrossi, A., and Landrini, M., 2003, "Numerical simulation of interfacial flows by smoothed particle hydrodynamics," Journal of Computational Physics, 191(2), pp. 448-475.

[5] Monaghan, J. J., 2012, "Smoothed Particle Hydrodynamics and Its Diverse Applications," Annual Review of Fluid Mechanics, 44(1), pp. 323-346.

[6] Price, D. J., 2012, "Smoothed particle hydrodynamics and magnetohydrodynamics," Journal of Computational Physics, 231(3), pp. 759-794.

[7] Rosswog, S., 2009, "Astrophysical smooth particle hydrodynamics," New Astronomy Reviews, 53(4-6), pp. 78-104.

[8] Monaghan, J. J., 2005, "Smoothed particle hydrodynamics," Reports on Progress in Physics, 68(8), p. 1703.

[9] Liu, G.-R., 2010, Meshfree methods: moving beyond the finite element method, CRC press.

[10] Springel, V., 2010, "Smoothed Particle Hydrodynamics in Astrophysics," Annual Review of Astronomy and Astrophysics, 48(1), pp. 391-430.

[11]Benz, W., 1990, "Smooth particle hydrodynamics: a review," The numerical modelling of nonlinear stellar pulsations, Springer, pp. 269-288.

[12] Monaghan, J., 1989, "On the problem of penetration in particle methods," Journal of Computational physics, 82(1), pp. 1-15.

[13] Wendland, H., 1995, "Piecewise polynomial, positive definite and compactly supported radial functions of minimal degree," Advances in computational Mathematics, 4(1), pp. 389-396.

[14]Dehnen, W., and Aly, H., 2012, "Improving convergence in smoothed particle hydrodynamics simulations without pairing instability," Monthly Notices of the Royal Astronomical Society, 425(2), pp. 1068-1082.

[15] Monaghan, J., and Kos, A., 1999, "Solitary waves on a Cretan beach," Journal of waterway, port, coastal, and ocean engineering, 125(3), pp. 145-155.

[16]Batchelor, G. K., 2000, An introduction to fluid dynamics, Cambridge university press.

[17]Dilts, G. A., 1999, "Moving-least-squares-particle hydrodynamics-I. Consistency and stability," International Journal for Numerical Methods in Engineering, 44(8), pp. 1115-1155.

[18] Panizzo, A., 2004, "Physical and numerical modelling of subaerial landslide generated waves," PhD Thesis, University of L'Aquila. 